\title{
Diffuse reflection of ultracold neutrons from low-roughness
}

\section{surfaces}

\section{Journal Article}

\section{Author(s):}

Atchison, F.; Daum, M.; Henneck, R.; Heule, S; Horisberger, M.; Kasprzak, M.; Kirch, K.; Knecht, A.; Kuzniak, M.; Lauss, B.; Mtchedlishvili, A.; Meier, M.; Petzoldt, G.; Plonka-Spehr, C.; Schelldorfer, R.; Straumann, U.; Zsigmond, G.

\section{Publication date:}

2010-04

\section{Permanent link:}

https://doi.org/10.3929/ethz-b-000027516

\section{Rights / license:}

In Copyright - Non-Commercial Use Permitted

\section{Originally published in:}

The European Physical Journal A 44(1), https://doi.org/10.1140/epja/i2010-10926-x 


\title{
Diffuse reflection of ultracold neutrons from low-roughness surfaces
}

\author{
F. Atchison ${ }^{1}$, M. Daum ${ }^{1}$, R. Henneck ${ }^{1, a}$, S. Heule ${ }^{1,2}$, M. Horisberger ${ }^{1}$, M. Kasprzak ${ }^{1,3}$, K. Kirch ${ }^{1}$, A. Knecht K $^{1,2}$, \\ M. Kużniak ${ }^{1,5, b}$, B. Lauss ${ }^{1}$, A. Mtchedlishvili ${ }^{1}$, M. Meier ${ }^{1}$, G. Petzoldt ${ }^{1}$, C. Plonka-Spehr ${ }^{4, c}$, R. Schelldorfer $^{1}$, \\ U. Straumann ${ }^{2}$, and G. Zsigmond ${ }^{1}$ \\ 1 Paul Scherrer Institut, PSI, Villigen, Switzerland \\ 2 University Zürich, Zürich, Switzerland \\ 3 Stefan Meyer Institut, Vienna, Austria \\ 4 Institut Laue Langevin, ILL, Grenoble, France \\ 5 Smoluchowski Institute of Physics, Jagiellonian University, Cracow, Poland
}

Received: 2 October 2009 / Revised: 18 December 2009

Published online: 10 March 2010 - (C) Società Italiana di Fisica / Springer-Verlag 2010

Communicated by R. Krücken

\begin{abstract}
We report a measurement of the reflection of ultracold neutrons from flat, large-area plates of different Fermi potential materials with low surface roughness. The results were used to test two diffuse reflection models, the well-known Lambert model and the micro-roughness model which is based on wave scattering. The Lambert model fails to reproduce the diffuse reflection data. The surface roughness $b$ and correlation length $w$, obtained by fitting the micro-roughness model to the data are in the range $1 \leq b \leq 3 \mathrm{~nm}$ and $10 \leq w \leq 120 \mathrm{~nm}$, in qualitative agreement with independent measurements using atomic force microscopy.
\end{abstract}

\section{Introduction}

Measuring the reflection of ultracold neutrons (UCN) from low-roughness reflectors allows testing diffuse reflection models. Neutron reflection is a specific example of the general problem of wave scattering from surfaces, a fundamental topic in physics since the development of geometrical optics. Diffuse (or non-specular) reflection was introduced in 1760 by Lambert [1] and still today the Lambert model (LM) is the baseline "diffuse" model implemented in most simulation packages. Later extensions to other frequency ranges and particles led to an immense broadening of the field, now encompassing many applications ranging from X-ray and neutron physics to topics in geology, the study of planet surfaces, sound and radar imaging, etc., see, e.g., $[2,3]$. Work relevant to reflection of X-rays and neutrons from rough surfaces has been performed over the last decades, see, e.g., [4-6].

UCN have relatively long wavelengths $(\lambda \geq 50 \mathrm{~nm})$ and are totally reflected under any angle of incidence from suitable materials. They allow, e.g., to measure the neutron lifetime and its electric dipole moment, important observables to test the standard model of particle physics

\footnotetext{
a e-mail: reinhold.henneck@psi.ch

b Now at Queens University, Kingston, Canada.

c Now at Gutenberg University, Mainz, Germany.
}

(see, e.g., [7-9]). Already in 1972, Steyerl [10] and Ignatovich [11] developed descriptions of UCN scattering from real surfaces, termed here micro-roughness (MR) models. Similar to the description of acoustic-wave scattering [12], they are based on distorted Green's functions and equivalent to the distorted-wave Born approximation treatment employed later $[5,6,13]$. The surface is treated as a perturbation of an ideally smooth plane causing diffuse scattering through diffraction and interference. The MR model is applicable to high-quality reflectors with surface roughness $b \ll \lambda$, leading to low values of the diffuse reflection probability $d^{\mathrm{MR}}$, typically below a few percent. It is most suitable for low-energy neutrons at angles less than the critical angle (as is the case of UCN). While in the second model used here, i.e. the Lambert model (LM), the reflected intensity follows a cosine distribution irrespective of the angle of incidence and depends only on $d^{\mathrm{LM}}$, $\mathrm{MR}$ diffuse reflection is characterized by the neutron energy, its angle of incidence and the roughness, correlation length and composition of the surface. This can lead to substantial deviations from LM-type reflection.

Both descriptions, the LM as well as the MR model, are formulated in terms of reflection probabilities and can be readily implemented into Monte Carlo trajectory tracking codes (such as GEANT4-UCN [14]), which allow the user to handle practically any geometry. For this reason we did not consider the transport operator description devel- 

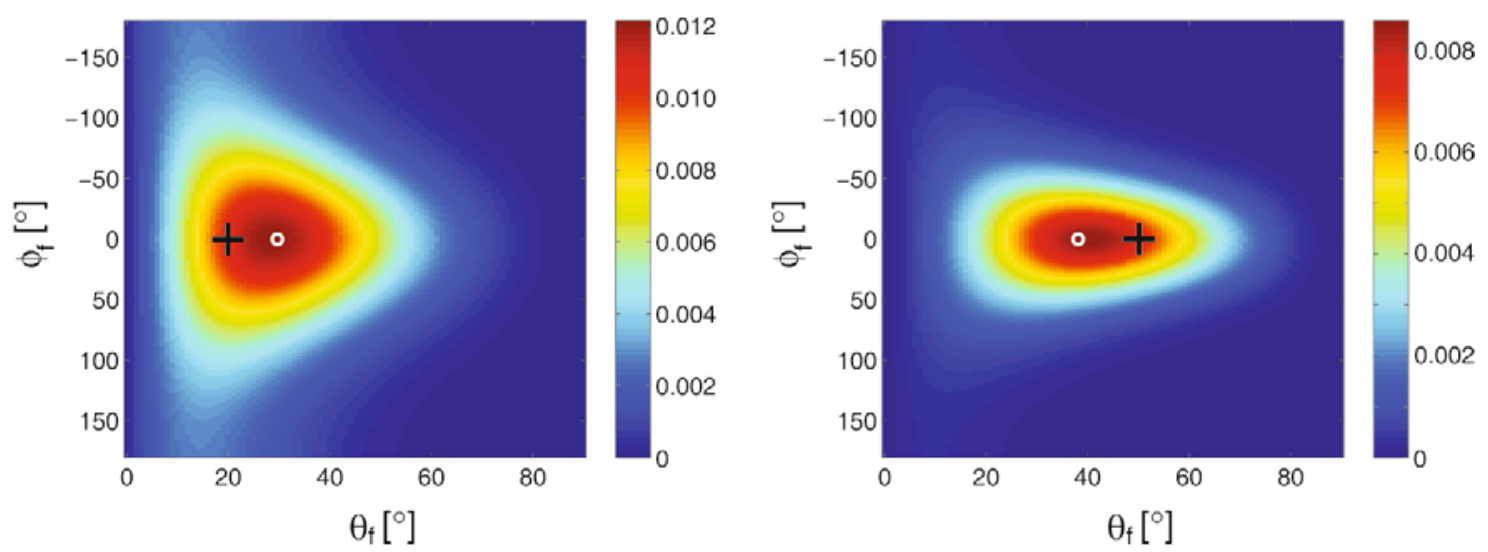

Fig. 1. (Colour on-line) Probability of non-specular surface reflection into unit solid angle, $I_{\mathrm{ns}}\left(\theta_{\mathrm{f}}, \Phi_{\mathrm{f}}\right)$, given by the MR model using $V_{\mathrm{F}}=220 \mathrm{neV}, E_{\mathrm{UCN}}=200 \mathrm{neV}, b=1 \mathrm{~nm}$ and $w=25 \mathrm{~nm}$. Left panel for $\theta_{\mathrm{i}}=20^{\circ}$, right panel for $\theta_{\mathrm{i}}=50^{\circ} ; \phi_{\mathrm{i}}=0^{\circ}$ in both cases. The crosses indicate the angles of incidence while the circles correspond to the 1st moments of $I_{\mathrm{ns}}$.

oped recently $[15,16]$, which delivers, e.g., transition probabilities between energy states or neutron transport integrated over specific simple geometries and would require major efforts for implementation into standard tracking codes.

The UCN interaction with a material surface is given by the coherent strong interaction, i.e. the Fermi (or optical) potential, $V_{\mathrm{F}}=V-i W$, where $V$ and $W$ depend on the nuclear properties of the surface (see, e.g., [17, 18]): $V=\frac{2 \pi \hbar^{2}}{m} \cdot N \cdot a, W=\frac{\hbar}{2} \cdot N \cdot \sigma \cdot v$. Here, $m$ and $v$ denote the neutron's mass and velocity, $N$ the atom number density, $a$ the bound coherent scattering length and $\sigma$ the loss cross-section. The real part, $V$, determines the height of the potential barrier, while the neutron loss is given by the imaginary part, $W$. Neutrons with velocity normal to the material surface, $v_{\text {norm }} \leq v_{\mathrm{c}}=\sqrt{2 \mathrm{~V} / \mathrm{m}}$ are totally reflected and designated UCN. For good, high-Fermi potential reflectors like $\mathrm{Be}$ or diamond-like carbon (DLC) $V$ is $\sim 250 \mathrm{neV}$ and the loss probability per bounce, $\eta=W / V$, is around $10^{-3}$ to $10^{-4}$ at room temperature, see, e.g, [18,19].

\section{The micro-roughness model}

The MR model of Steyerl [10,13] calculates diffuse reflection from microscopically rough surfaces using a Gaussian correlation function. The latter is standard in the literature for describing surfaces without periodic structure, for the treatment of other surface roughness models see, e.g. [4-6]. The probability of non-specular reflection of an UCN with wave vector $k$, incident at $\theta_{\mathrm{i}}\left(\phi_{\mathrm{i}}=0\right)$ to the normal of a surface with rms roughness $b$ and lateral correlation length $w$ is given by

$$
\begin{aligned}
& I_{\mathrm{ns}}\left(\theta_{\mathrm{f}}, \Phi_{\mathrm{f}}\right)=\frac{k_{\mathrm{c}}^{4} \cdot(b w)^{2}}{8 \pi \cdot \cos \theta_{\mathrm{i}}}\left|S\left(\theta_{\mathrm{i}}\right)\right|^{2}\left|S\left(\theta_{\mathrm{f}}\right)\right|^{2} \\
& \times \exp \left[-\frac{(w k)^{2}}{2}\left(\sin ^{2} \theta_{\mathrm{i}}+\sin ^{2} \theta_{\mathrm{f}}-2 \sin \theta_{\mathrm{i}} \sin \theta_{\mathrm{f}} \cos \Phi_{\mathrm{f}}\right)\right],
\end{aligned}
$$

where, e.g., $S\left(\theta_{\mathrm{i}}\right)=2 \cos \theta_{\mathrm{i}} /\left(\cos \theta_{\mathrm{i}}+\left(\cos ^{2} \theta_{\mathrm{i}}-k_{\mathrm{c}}^{2} / k^{2}\right)^{1 / 2}\right)$. Here, $k_{\mathrm{c}}=\sqrt{\frac{2 m V}{\hbar^{2}}}$ is the critical wave vector for total reflection and $\theta_{\mathrm{f}}, \phi_{\mathrm{f}}$ are the angles after reflection.

This approach is valid for $2 b k_{\mathrm{c}} \leq 1,2 b k \cos \theta_{\mathrm{i}} \leq 1$ and $2 b k \cos \theta_{\mathrm{f}} \leq 1$, restricting its validity to highly polished surfaces with $b \leq 5 \mathrm{~nm}$ for UCN. The non-specular component $I_{\mathrm{ns}}\left(\theta_{\mathrm{f}}, \Phi_{\mathrm{f}}\right)$ is sharply peaked about the direction of specular reflection for long correlation lengths $\left((k w)^{2} \gg 1\right)$, while for shorter correlation lengths, i.e. $(k w)^{2} \ll 1$, as in our case, it is typically a broad distribution with FWHM of $\sim 50^{\circ}$ in $\theta_{\mathrm{f}}, \phi_{\mathrm{f}}$; the average direction depends on $\theta_{\mathrm{i}}$ and can differ significantly from the specular direction. This is illustrated in fig. 1 for two different angles of incidence.

From eq. (1) one obtains the total probability of diffuse reflection for a given energy spectrum $f\left(E, \theta_{\mathrm{i}}\right)$,

$$
d^{\mathrm{MR}}(b, w, V)=\int f\left(E, \theta_{\mathrm{i}}\right) I_{\mathrm{ns}}\left(\theta_{\mathrm{f}}, \Phi_{\mathrm{f}}\right) \mathrm{d} E \mathrm{~d} \theta_{\mathrm{i}} \mathrm{d} \Omega_{\mathrm{f}} .
$$

It increases with $b, w, V$ and $E$ (for $E \cdot \cos ^{2} \theta_{\mathrm{i}}<V$ ), while it decreases with angle of incidence. It is considerably enhanced for $E \cdot \cos ^{2} \theta_{\mathrm{i}} \leq V$ and a good test of the MR model can be performed with UCN.

There are, to date, no stringent experimental tests. Evidence for the MR model by ref. [20] is considered weak since the fitted surface parameters were at the limit of applicability and no independent measurements of these parameters available. Experiments [21-23] do not allow for a test, since they record either a very low $[21,22]$ or an unknown fraction of diffusely reflected neutrons [23]. Altarev et al. [24] applied the method of [25] to measure both, the loss probability due to absorption and inelastic up-scattering and the diffuse reflection probability for a $3.4 \mathrm{~m}$ long, beryllium-coated guide. Unfortunately, no characterization of the surfaces was performed to relate the extracted quantities to an independent analysis of the surface roughness.

Improved knowledge about diffuse reflection is necessary for the design of UCN optical instrumentation, i.e. transport guides, microscopes and spectrometers (see, 


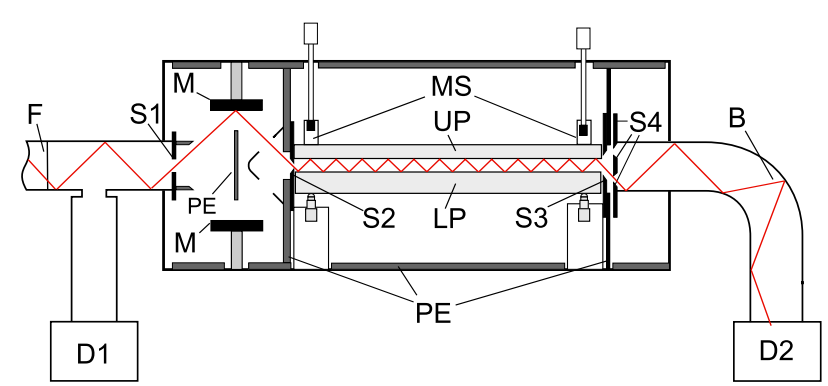

Fig. 2. (Colour on-line) Experimental setup; for details see text. The thin line (in red on-line) represents a specularly reflected neutron trajectory passing through the system.

e.g., $[17,18])$. Guide transmission (typically over distances of $5 \mathrm{~m}$ ) depends mainly on three factors, i) the loss per bounce, $\eta=W / V$, ii) the Fermi potential and iii) the amount of diffuse reflection. Diffuse reflection will lead to diffusion-like transport (i.e. lower effective flow velocities) and enhanced loss due to a higher number of wall collisions, especially as the UCN can travel backwards. For neutrons of total energy $E>V$ the possible change in direction will lead to loss when $\theta_{\mathrm{i}}<\theta_{\mathrm{c}}\left(E \cos ^{2} \theta_{\mathrm{c}}=V\right)$. Measured values for the transmission of practical guides are $\leq 0.85$ per meter of length for $66 \mathrm{~mm}$ diameter $[17,18,24]$. Since the number of reflections per meter is $\sim 6$ from simulation, it is obvious that diffusivity dominates transport loss.

\section{Experiment}

Our experiment (see fig. 2) is a significantly improved version of [23] and measures the transmission of a collimated UCN beam through a gap defined by two flat sample plates (for details see [26]). The gap height defines the number of reflections. The diffuse reflection component leads to a decrease in transmission which depends on the number of reflections and thus on the gap height.

After passing a thin $\mathrm{Al}$ foil $(\mathrm{F})$ the $\mathrm{UCN}$ entered the slit $\mathrm{S} 1$, which in combination with the Ni-coated mirrors $\mathrm{M}$ and the slit $\mathrm{S} 2$ collimated the beam entering the plate pair at $\theta_{\mathrm{i}}=(51 \pm 3)^{\circ}$. From simulation we know that angles around $50^{\circ}$ are most frequently encountered in typical UCN guides. The upper plate (UP) could be moved under vacuum conditions via micrometer screws (MS) with a reproducibility of $5 \mu \mathrm{m}$. For a given S2 slit height (usually $2.5 \mathrm{~mm}$ ) the gap height $s$ was varied between 3.5 and $6.0 \mathrm{~mm}$. Correspondingly, the average number of specular reflections changed between $n=116$ and $n=68\left(n=\cot \theta_{\mathrm{i}} \cdot L / s, L=500 \mathrm{~mm}\right.$ the plate length $)$. The angular acceptance was restricted by slits $\mathrm{S} 3\left( \pm 6^{\circ}\right.$ horizontally) and S4 (double slit with separation $12 \mathrm{~mm}$, $\left.40^{\circ} \leq \theta_{\mathrm{f}} \leq 60^{\circ}\right)$. All slits were made from either $\mathrm{Ti}$ $(V=-48 \mathrm{neV})$ or $4 \%$ borated $\mathrm{Al}$ in order to minimize scattering effects. Stray background was found to be negligible from a measurement with a tightly fitting UCN absorber (polyethylene, PE) between the plates. The positions of all relevant elements were measured on a $3 \mathrm{D}$ measuring machine with an accuracy of several microns. The plate settings were checked independently $( \pm 5 \mu \mathrm{m})$ during the experiment by placing precision pins between the plates. The detectors D1, D2 were ${ }^{3} \mathrm{He}$ gas counters, shielded with $\mathrm{Cd}$ and borated $\mathrm{PE}$.

The experiment was performed at the PF2 UCN beamline at ILL [27]. Typical count rates (background rates) were $\sim 19(0.07) \mathrm{cps}$ for D1 and $\sim 0.2$ to $\sim 13(0.015) \mathrm{cps}$ for D2. For separation of the two reflection components we took data for 4 configurations (see fig. 5): 1) "parallel"; labeled by the plate distance at entrance and exit, e.g. $5 \mathrm{~mm} / 5 \mathrm{~mm}$ was labeled 5/5. 2) "convergent"; the top plate was tilted such that the plate spacing at entrance was larger than at exit, e.g. $5 \mathrm{~mm} / 3 \mathrm{~mm}$ was labeled $5 / 3$. Convergent configurations are very sensitive to non-specular reflection, since specular trajectories are all reflected backwards for sufficiently large tilt angles $\zeta \geq \zeta_{0}$. For a front gap width of $5 \mathrm{~mm} \zeta_{0} \sim 0.15^{\circ}$, see fig. 5 . D2 counts recorded for such settings must originate from diffuse reflection. 3) "Strip" ("S"); plates parallel, but the double-slit S4 removed and replaced by a $12 \mathrm{~mm}$ high, horizontal Al strip, similar in size to the central part of S4. This configuration accepted more UCN diffusely reflected into (mostly smaller) angles which would otherwise have hit the top and bottom part of S4. 4) "Open" ("O"); plates parallel, but $\mathrm{S} 4$ removed. This provided the largest admixture of diffuse reflections.

The spectrum of the velocity component along the guide axis (delivered by the guide only) was measured by replacing the experiment by a time-of-flight setup with chopper [28]. From this, absolute velocities were calculated using the simulated angular distribution accepted by the experiment. The absolute velocity spectrum is consistent with attenuation measurements, where we placed $\mathrm{PE}$ foils of different thickness (between 0.01 and $0.25 \mathrm{~mm}$ ) in front of S2 and observed a foil thickness dependence of D2 in agreement with the velocity spectrum measured with the chopper and with the correct $1 / v$ proportionality of the loss cross-section.

Vibration measurements on the vacuum chamber showed the maximum amplitude and velocity (about $0.4 \mu \mathrm{m}$ and $4.5 \cdot 10^{-4} \mathrm{~m} / \mathrm{s}$ ) at $16 \mathrm{~Hz}$, well below the sample collision frequency of about $1 \mathrm{kHz}$, such that acceleration effects are averaged out and are negligible.

\subsection{Samples and sample characterization}

We wanted to test samples representative of what can be used for UCN guides. Floatglass is easily available and exhibits excellent roughness values without additional polishing (for highly polished, but small-size samples, see [21]). Similarly, the handling and cleanliness conditions were as in a typical experiment, i.e. transport of the plates wrapped in silk paper and transfer in air. The sample plates (see table 1) were $500 \mathrm{~mm}$ long, 54 to $70 \mathrm{~mm}$ wide and 8 to $15 \mathrm{~mm}$ thick. With the exception of the Ni-replika (Ni-rep, same as those used in [23]) and the stainless-steel plates (SS), all samples were made from floatglass, either left untreated (FG), polished (FG-pol), 
Table 1. Results, ordered according to the MR model fitted roughness, $b_{\text {fit }}^{\mathrm{MR}}$ (see also text): 1) plate identification, 2) averaged $\mathrm{AFM}$ surface roughness $\left.b_{\mathrm{av}}^{\mathrm{AFM}}, 3\right)$ intrinsic AFM surface roughness $\left.b_{\mathrm{min}}^{\mathrm{AFM}}, 4\right)$ averaged AFM correlation length $\left.w^{\mathrm{AFM}}, 5\right) \mathrm{MR}$ fitted roughness $\left.b_{\mathrm{fit}}^{\mathrm{MR}}, 6\right) \mathrm{MR}$ fitted correlation length $\left.w_{\mathrm{fit}}^{\mathrm{MR}}, 7\right) \mathrm{MR}$ fitted diffusity $\left.d^{\mathrm{MR}}, 8\right)$ LM fitted diffusity $\left.d^{\mathrm{LM}}, 9\right)$ ratio of $\chi_{\text {dof }}^{2}$-values, $\left.\chi_{\mathrm{LM}}^{2} / \chi_{\mathrm{MR}}^{2}, 10\right)$ transmission $T^{\text {full }}$ of a $5 \mathrm{~m}$ guide, $66 \mathrm{~mm}$ inner diameter for the full energy spectrum calculated with the MR model; $T^{\mathrm{UCN}}$ with an upper energy limit of $250 \mathrm{neV}$.

\begin{tabular}{|c|c|c|c|c|c|c|c|c|c|}
\hline Plate id & $\begin{array}{c}b_{\mathrm{av}}^{\mathrm{AFM}} \\
{[\mathrm{nm}]}\end{array}$ & $\begin{array}{c}b_{\mathrm{min}}^{\mathrm{AFM}} \\
{[\mathrm{nm}]}\end{array}$ & $\begin{array}{c}w^{\mathrm{AFM}} \\
{[\mathrm{nm}]}\end{array}$ & $\begin{array}{l}b_{\mathrm{fit}}^{\mathrm{MR}} \\
{[\mathrm{nm}]}\end{array}$ & $\begin{array}{l}w_{\mathrm{fit}}^{\mathrm{MR}} \\
{[\mathrm{nm}]}\end{array}$ & $\begin{array}{c}d^{\mathrm{MR}} \\
{[\%]}\end{array}$ & $\begin{array}{c}d^{\mathrm{LM}} \\
{[\%]}\end{array}$ & $\chi_{\mathrm{LM}}^{2} / \chi_{\mathrm{MR}}^{2}$ & $T^{\text {full }}\left(T^{\mathrm{UCN}}\right)$ \\
\hline DLC & $0.3(1)$ & 0.1 & $37(5)$ & $0.9(1)$ & $34(13)$ & $1.4(3)$ & $0.71(1)$ & 90 & $0.70(0.80)$ \\
\hline $\mathrm{FG}$ & $1.0(2)$ & 0.1 & $37(6)$ & $0.9(1)$ & $96(35)$ & $0.8(1)$ & $0.31(3)$ & 6 & $0.49(0.66)$ \\
\hline $\mathrm{Ni}-\mathrm{V}$ & $0.6(1)$ & 0.2 & $23(4)$ & $1.0(1)$ & $15(4)$ & $1.0(2)$ & $0.99(1)$ & 310 & $0.75(0.81)$ \\
\hline FG-pol & $* *$ & $* *$ & $* *$ & $1.0(1)$ & $126(30)$ & $1.0(2)$ & $0.11(3)$ & 5 & $0.47(0.64)$ \\
\hline Ni-rep & $1.5(1)$ & 0.6 & $39(2)$ & $1.1(1)$ & $22(5)$ & $1.7(5)$ & & & $0.68(0.78)$ \\
\hline FG-HF & 5.1 & & & $1.8(4)$ & $92(40)$ & $3.0(4)$ & $0.87(5)$ & 334 & $0.36(0.47)$ \\
\hline SS & $2.5(5)$ & 0.9 & $37(5)$ & $2.6(1)$ & $20(2)$ & $6.9(1.3)$ & $3.35(12)$ & 126 & $0.34(0.44)$ \\
\hline $\mathrm{Ni}-\mathrm{Mo}$ & $1.5(1)$ & 1.1 & $23(1)$ & $2.4(3)$ & $12(2)$ & $5.0(1.3)$ & $3.31(9)$ & 6 & $0.43(0.59)$ \\
\hline $\mathrm{Ni}-8 / 9$ & $1.6(2)$ & 1.2 & $32(3)$ & $2.7(1)$ & $25(1)$ & $10.8(2.9)$ & & & $0.30(0.47)$ \\
\hline $\mathrm{Ni}-5 / 10$ & $* *$ & $* *$ & $* *$ & $3.1(1)$ & $25(1)$ & $14.6(3.9)$ & & & \\
\hline
\end{tabular}

** Plates too thick for AFM measurement.

etched with hydrofluoric acid (FG-HF; 30 min in 2\% HF) or coated with $\mathrm{Ni}$ (100\% natural), Ni-V (7 at-\%), Ni-Mo (7 at-\%) or diamond-like carbon (DLC). In general the plates displayed a single bend over the length of $500 \mathrm{~mm}$, with amplitudes of $\sim 0.05 \mathrm{~mm}(\mathrm{FG}), \sim 0.1 \mathrm{~mm}(\mathrm{SS})$ and $\sim 0.2 \mathrm{~mm}$ (Ni-rep). Plate curvature introduces unwanted angle transformations upon reflection which can modify the number of reflections. Its effect can be reduced by combining a "concave" plate with a "convex" one; e.g. if the two plates have exactly the same curvature the angle change introduced by the reflection from one plate is fully compensated by the subsequent reflection from the other plate. We selected matching plates so as to minimize the width variation along the gap; the residual curvature effect was calculated and considered as a (negligible) error contribution, see sect. 5. The $\mathrm{Ni}$ coatings were produced with rf magnetron sputtering, by PSI (Ni-8/9, $400 \mathrm{~nm}$ thick; Ni-5/10, $1000 \mathrm{~nm}$ thick; Ni-Mo, $400 \mathrm{~nm}$ thick) and by S-DH GmbH, Heidelberg, Germany (Ni-V, $1000 \mathrm{~nm}$ thick). The DLC coatings (250 nm thick) were produced with ion beam sputtering by Fraunhofer IWS, Dresden, Germany. A $s p^{3}$ fraction of about 0.3 was extracted from X-ray Photon Spectroscopy (XPS), X-ray reflectivity and slow neutron reflectivity measurements. The SS plates were precision-machined, hand-polished and electro-polished. The uncoated plates were cleaned with isopropanol, followed by rinsing with high-purity distilled water. Before installation in the chamber the plates were blown with air spray.

Roughness characterization of the surfaces was performed by atomic force microscopy (AFM, Veeco DI-3100) which is well suited since the scan length can be adapted to cover the UCN wavelength region. Employing X-ray reflectometry at grazing incidence would call for sample flatness over several tens of centimetres, i.e. large, optically flat plates. We used three scan sizes $\left(1 \times 1 \mu \mathrm{m}^{2}\right.$,
$10 \times 10 \mu \mathrm{m}^{2}$ and $\left.100 \times 100 \mu \mathrm{m}^{2}\right)$ with lateral resolutions of about 2, 20, $200 \mathrm{~nm}$. Each scan consisted of $512 \times 512$ data points, from which the rms roughness and correlation length were calculated. Scans at about 10 positions were evaluated on each plate. The rms roughnesses $b_{\mathrm{av}}^{\mathrm{AFM}}$ and correlation lengths $w^{\mathrm{AFM}}$ given in table 1 are averages over the individual $1 \times 1 \mu \mathrm{m}^{2}$ scan values and the uncertainties given correspond to the rms deviation. An exception was FG-HF, where etching by HF had created a "pitted" structure with isolated pits of typically 5 micron size (cf. also e.g. [29]). Here, $b_{\mathrm{av}}^{\mathrm{AFM}}$ and $w^{\mathrm{AFM}}$ were extracted from the $100 \times 100 \mu \mathrm{m}^{2}$ scans. The variation of $b^{\mathrm{AFM}}$ for the different measurement positions was up to a factor 4 , related to occasional dust particles, coating defects, etc. We therefore also extracted an "intrinsic" roughness value, $b_{\min }^{\mathrm{AFM}}$, corresponding to the minimum roughness and attributed to a surface not affected by such defects. These values ranged from $\sim 0.1 \mathrm{~nm}(\mathrm{FG})$ to $\sim 1.2 \mathrm{~nm}(\mathrm{Ni}-8 / 9)$, see table 1 . Figure 3 shows two examples of such "clean" $1 \times 1 \mu \mathrm{m}^{2}$ scans.

The Fermi potentials were measured by cold neutron reflection with the Narziss instrument, SINQ, PSI [30] in a way similar to that described in ref. [28]. They are consistent with the coating compositions and scattering lengths $220 \pm 10 \mathrm{neV}(\mathrm{Ni}-\mathrm{V}), 235 \pm 10 \mathrm{neV}(\mathrm{Ni}-\mathrm{Mo}), 239 \pm 10 \mathrm{neV}$ (Ni), $220 \pm 10 \mathrm{neV}$ (DLC) and $84 \pm 8 \mathrm{neV}$ (FG).

\section{Analysis}

In a first step we followed the simple approach of refs. $[22,23,31]$ which neglects diffuse reflection. The D2 count rates were background corrected, normalized to the (background corrected) D1 count rates and plotted as a function of the average number of specular reflections, $n$. 

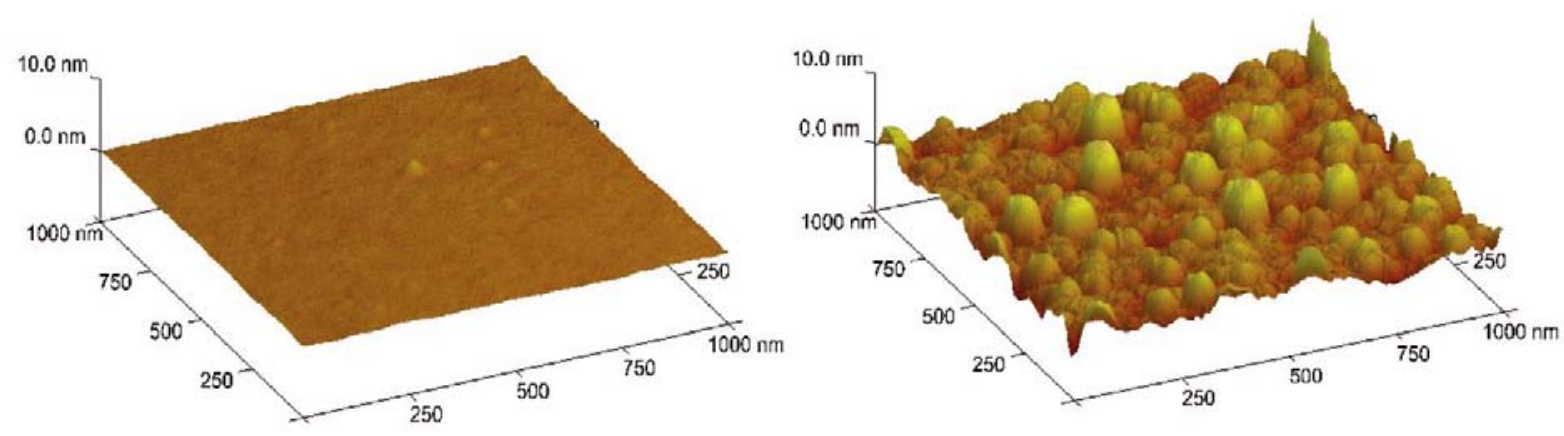

Fig. 3. (Colour on-line) "Clean" $1 \times 1 \mu \mathrm{m}$ AFM scans of FG (left) and of Ni-8/9 (right). The vertical scale is from $-10 \mathrm{~nm}$ to $+10 \mathrm{~nm}$.

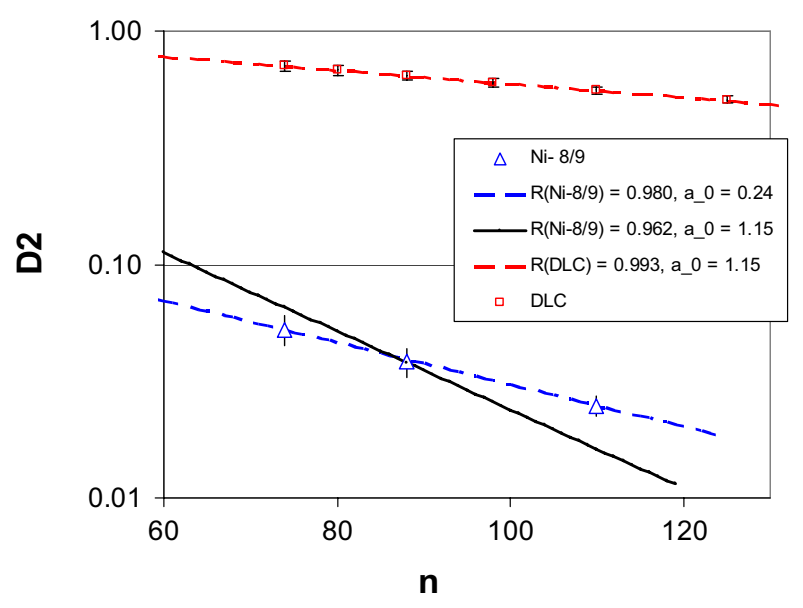

Fig. 4. (Colour on-line) Normalized D2 count rate versus number of specular reflections $n$ : DLC (squares) compared to $\mathrm{Ni}-$ $8 / 9$ (open triangles). The dashed lines were obtained by fitting both, $R_{\mathrm{s}}$ and $a_{0}$ to eq. (3), while the solid line is a fit to Ni-8/9 with $a_{0}$ constrained to $a_{0}=1.15$ (as for DLC).

The distribution of the number of specular reflections for the accepted phase space was calculated using GEANT4UCN [14], taking into account gravitation and the details of the setup and of the beam energy distribution. As an example we show in fig. 4 two D2 $(n)$ curves, together with the respective "specular" fit functions

$$
\mathrm{D} 2(n)=a_{0} R_{\mathrm{s}}^{n},
$$

where $R_{\mathrm{S}}$ is the probability of specular reflection per wall collision.

The normalization parameter $a_{0}$ corresponds to D2 $(n=0)$, i.e. to the case of no reflection, which must be identical for any plate pair, if the setup and beam properties have not changed. Using this universal value (approximated by the value determined from a fit to a closeto-perfect mirror like DLC, $a_{0} \sim 1.15$ ) it can readily be seen that the reflectivity of less perfect mirrors like Ni$8 / 9$ cannot be described by a purely specular approach and that diffuse reflection has to be considered. It should be pointed out that the reflectivities in refs. $[22,23,31]$ were obtained by fitting both, $R_{\mathrm{s}}$ and $a_{0}$ to the individual
D2 $(n)$ curves. As shown in fig. 4 this can lead to a significant over-estimation of the value of $R_{\mathrm{s}}$ : e.g. applying the proper constraint on $a_{0}$ changed the value of $R_{\mathrm{s}}$ for $\mathrm{Ni}-8 / 9$ from 0.98 to 0.962 .

In the final analysis all configurations were fully simulated in GEANT4-UCN [14] with both the MR and LM models implemented. In order to determine the values of $b^{\mathrm{MR}}$ and $w^{\mathrm{MR}}$ of the MR model which correspond to the different sample plates, simulated D2 data were calculated for the relevant part of the parameter space $\left(0 \leq b^{\mathrm{MR}} \leq 4 \mathrm{~nm}, 0 \leq w^{\mathrm{MR}} \leq 200 \mathrm{~nm}\right)$. In a similar fashion this was done for the LM model, however, only using one parameter, the fraction of diffuse reflection $d^{\mathrm{LM}}$. The loss factor used $\left(\eta=(10 \pm 9) \cdot 10^{-4}\right)$ reflects the large spread in measured values $[18,19,24]$. The experimental D2 data (background corrected, normalized to D1) were fitted to the interpolated simulated D2 data, employing a free normalization parameter $f$. Since $f$ should be a constant, any larger spread of $f$ for the different samples is an indication for the inadequacy of the model used. For MR $f$ indeed displayed a narrow distribution while for LM $f$ varied by up to a factor 50 in extreme cases (hence we do not show results for Ni-rep, Ni 8/9, Ni 5/10). Although this indicates already a failure of the LM analysis, we nevertheless quote its results, however, using the average value of $f$ extracted from the MR model fits.

The sensitivity to various reflection models is given in fig. 5, where we have plotted the simulated transmission through the system as a function of the tilt angle $\zeta$ for the purely specular case, for a MR configuration with $b=1 \mathrm{~nm}, w=25 \mathrm{~nm}$ and for two LM cases with $d^{\mathrm{LM}}=1 \%, 5 \%$. Figure 5 demonstrates that the experiment is sensitive to diffuse reflection and - moreoverallows discriminating between different diffuse reflection models.

\section{Results and discussion}

Table 1 gives the results for $b_{\text {fit }}^{\mathrm{MR}}, w_{\text {fit }}^{\mathrm{MR}}, d^{\mathrm{MR}}$ and $d^{\mathrm{LM}}$. The uncertainties include the statistical errors and an estimate of the systematic uncertainties due to a) Fermi potentials, b) loss factors and c) plate curvatures. Inclusion of these effects raised the total errors by a factor 3 to 5 , the dominant contribution caused by b). Figure 6 shows 


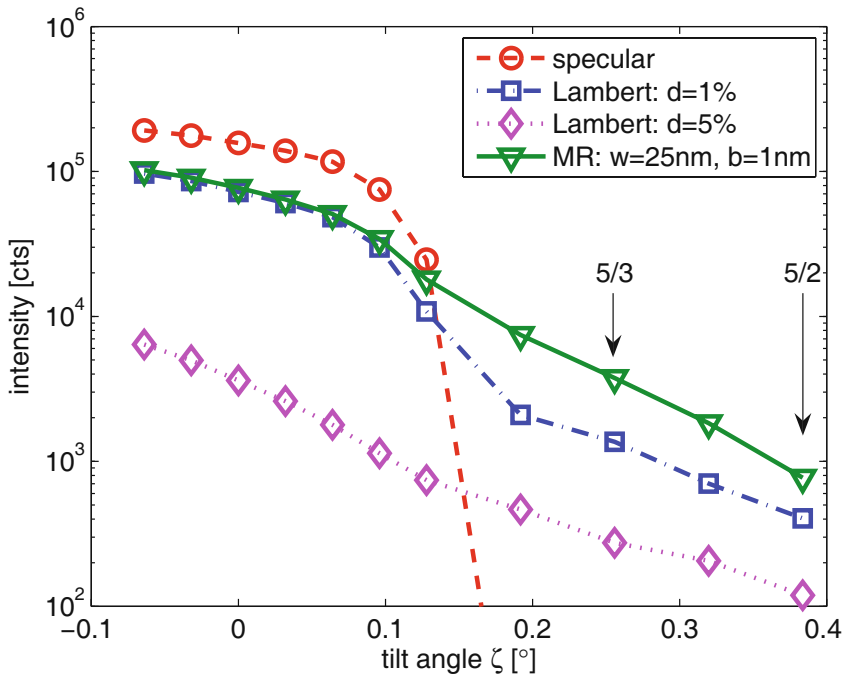

Fig. 5. (Colour on-line) Simulated number of neutrons transmitted through the system (using DLC coating here) as a function of the tilt angle $\zeta$, starting from $10^{6} \mathrm{UCN}$ at S2 (colour on-line). Purely specular reflection (open circles) is compared with partially diffuse reflection using the LM with $d^{\mathrm{LM}}=1 \%$ (squares) and $5 \%$ (diamonds) and the MR with $b=1 \mathrm{~nm}$, $w=25 \mathrm{~nm}$ (triangles). The lines between the points are to guide the eye. The normally used converging configurations $5 / 3$ and $5 / 2$ are also indicated.

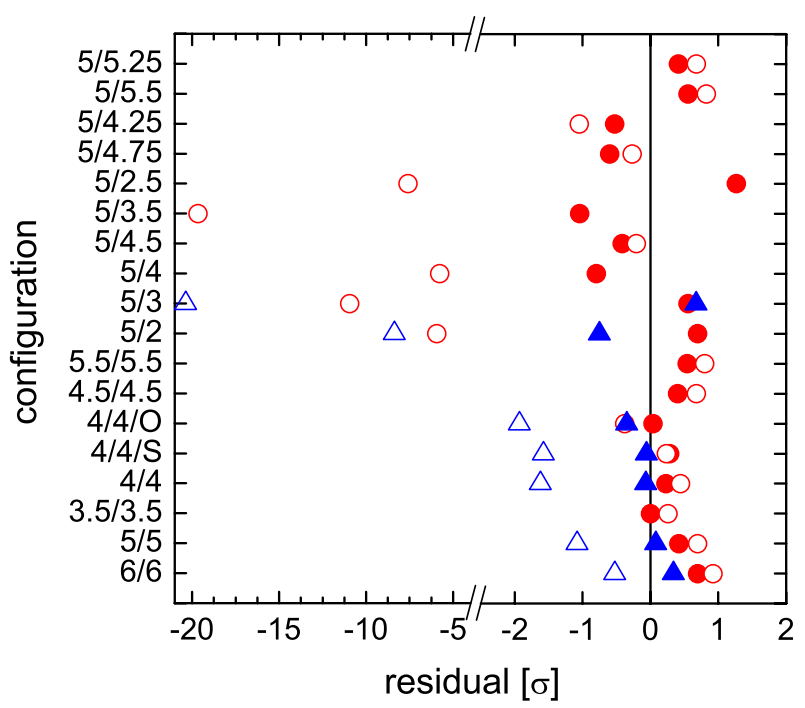

Fig. 6. (Colour on-line) Model results minus experimental data for the different configurations (plotted in units of the rms value $\sigma$ ) obtained with DLC (filled circles: MR, open circles: LM) and with $\mathrm{Ni}-\mathrm{V}$ (filled triangles: MR, open triangles: LM). Please note the change in scale on the horizontal axis.

the comparison between experiment and model results for DLC and Ni-V. Both models describe the parallel and close-to-parallel configurations about equally well. The LM fails for sufficiently convergent configurations, leading to $\chi_{\text {dof }}^{2}$-values (per degree of freedom) of 40 (DLC) and $99(\mathrm{Ni}-\mathrm{V})$. By contrast, the MR model fits the full data sets well, with $\chi_{\mathrm{dof}^{-}}^{2}$-values $(0.44$ for DLC, 0.32 for $\mathrm{Ni}-\mathrm{V})$ indicating some over-estimation of systematic errors. A similar behavior is observed for all samples, as reflected by the ratio of $\chi_{\mathrm{dof}}^{2}$-values, $\chi_{\mathrm{LM}}^{2} / \chi_{\mathrm{MR}}^{2}$, in table 1 .

The ordering of table 1 according to $b_{\text {fit }}^{\mathrm{MR}}$ is consistent with the intrinsic AFM roughness, $b_{\min }^{\mathrm{AFM}}$. This finding supports the concept of the surface being a superposition of clean, blemish-free areas (characterized by $b_{\min }^{\mathrm{AFM}}$ ) with occasional peaks caused by dust particles and coating defects. Note, that AFM may see structures (dust particles, etc.) which can be transmitted by $\mathrm{UCN}$ for $V<E$ and are thus not measured with the same sensitivity. Vice versa, UCN reflection is sensitive to sub-surface density inhomogeneities, whereas AFM is not. Considering this, the consistency between AFM-derived and UCN-derived surface parameters is satisfactory (and on a level similar to that obtained by comparing X-ray reflection with scanning tunneling microscopy, see e.g. [32]).

Column 10 in table 1 shows the transmission through a typical guide tube (5 m length, $66 \mathrm{~mm}$ diameter), calculated with MR for the PF2 beam energy spectrum ( $T^{\text {full }}$, containing about $70 \%$ neutrons above $250 \mathrm{neV}$ ) and with a cutoff at $250 \mathrm{neV}\left(T^{\mathrm{UCN}}\right)$. The predictions for DLC, Ni-V, Ni-rep are significantly higher than those reported in previous studies $[17,18,24]$ and highlight the necessity to prepare low-roughness surfaces. Concerning the use of tubes in practice, we measured the AFM roughness of the internal surface of "as delivered" glass tubes to be on a level of 2 to $3 \mathrm{~nm}$ over a length scale of 1 micrometer. Quartz tubes and polished stainless-steel tubes coated with ${ }^{58} \mathrm{Ni}$ were reported to be on a similar level [33].

In conclusion, we have performed a first systematic test of UCN reflection from large-area, high-quality surfaces over an extended range of surface roughness (1 to $3 \mathrm{~nm}$ ) and Fermi potentials (90 to $250 \mathrm{neV}$ ). In contrast to the Lambert model the micro-roughness model is able to reproduce the diffuse reflection data. The fitted surface parameters are in reasonable agreement with the results from independent AFM measurements.

This work was performed at PSI and ILL. The hospitality of ILL is gratefully acknowledged. We are indebted to B. Gubser (Optico AG, Sevelen, Switzerland) H. Häse (S-DH GmBH, Heidelberg) and P. Gawlitza (IWS Dresden) for providing the polished FG plates and the Ni-V and DLC coatings. We thank T. Brenner, P. Böhler, U. Bugmann, M. Dänzer, T. Gahl, M. Giebultowski, H. Grimmer, M. Müller, M. Schneider and P. Thomsen-Schmidt for substantial contributions. The work was supported by the Swiss National Science Foundation under grant No. 200021-105400.

\section{References}

1. J.H. Lambert, Photometria sive de mensure et gradibus luminis colorum et umbra (Eberhard Klett, Augsburg, 1760).

2. P. Beckmann, A. Spizzichino, The Scattering of Electromagnetic Waves from Rough Surfaces (Artech House, Norwood, MA, 1987). 
3. A.G. Voronovich, Wave Scattering from Rough Surfaces, edited by L.M. Brekhovskikh, L.B. Felsen, H.A. Haus, Springer Ser. Wave Phenom., Vol. 17 (1998).

4. L. Nevot, P. Croce, Rev. Phys. Appl. 15, 761 (1980).

5. S.K. Sinha, E.B. Sirota, S. Garoff, H.B. Stanley, Phys. Rev. B 38, 2297 (1988).

6. R. Pynn, Phys. Rev. B 45, 602 (1992).

7. I.B. Khriplovich, S.K. Lamoreaux, CP Violation without Strangeness: Electric Dipole Moments of Particles, Atoms and Molecules (Springer-Verlag, 1997) ISSN 0172-5998.

8. H. Abele, Prog. Part. Nucl. Phys. 60, 1 (2008).

9. M. Pospelov, A. Ritz, Ann. Phys. (N.Y.) 318, 119 (2005).

10. A. Steyerl, Z. Phys. 254, 169 (1972).

11. V.K. Ignatovich, JINR, Dubna, Report P4-7055 (1973).

12. P.M. Morse, U. Ingard, Theoretical Acoustics (Princeton University Press, Princeton NJ, 1968).

13. A. Steyerl, S.S. Malik, L.R. Iyengar, Physica B 173, 47 (1991).

14. F. Atchison, T. Brys, M. Daum, P. Fierlinger, A. Fomin, R. Henneck, K. Kirch, M. Kuzniak, A. Pichlmaier, Nucl. Instrum. Methods A 552, 513 (2005) (The code is maintained at PSI and can be obtained from G. Zsigmond.)

15. A.E. Meyerovich, A. Stephaniants, Phys. Rev. B 60, 9129 (1999).

16. R. Adhikari, Y. Cheng, A.E. Meyerovich, V.V. Nesvizhevskii, Phys. Rev. A 75, 063613 (2007).

17. R. Golub, D.J. Richardson, S.K. Lamoureaux, Ultra-Cold Neutrons (Adam Hilger, Bristol, Philadelphia and New York, 1991) ISBN 0-7503-0115-5.

18. V.K. Ignatovich, The Physics of Ultracold Neutrons (Clarendon Press, Oxford, 1990) ISBN 0-19-851015-2.
19. F. Atchison, T. Brys, M. Daum, P. Fierlinger, A. Foelske, M. Gupta, R. Henneck, S. Heule, M. Kasprzak, K. Kirch, R. Koetz, M. Kuzniak, T. Lippert, C.F. Meyer, F. Nolting, A. Pichlmaier, D. Schneider, P. Siemroth, U. Straumann, Phys. Lett. B 625, 19 (2005).

20. K.J. Kügler, W. Paul, U. Trinks, Z. Phys. B: Condens. Matter 39, 361 (1980).

21. V.V. Nesvizhevsky, Nucl. Instrum. Methods A 557, 576 (2006).

22. V.V. Nesvizhevsky, G. Pignol, K.V. Protasov, G. Quemener, Nucl. Instrum. Methods A 578, 435 (2007).

23. C. Plonka, P. Geltenbort, T. Soldner, H. Haese, Nucl. Instrum. Methods A 578, 450 (2007).

24. I. Altarev, A. Frei, P. Geltenbort, E. Gutsmiedl, F.J. Hartmann, A.R. Mueller, S. Paul, C. Plonka, D. Tortorella, Nucl. Instrum. Methods A 570, 101 (2007).

25. V.K. Ignatovich et al., JINR, Dubna, Report P3-82-811 (1982).

26. S. Heule, PhD Thesis, University of Zurich, unpublished (2008).

27. ILL yellow book, http://wwww.ill.fr/YellowBook/PF2/.

28. F. Atchison, B. Blau, M. Daum, P. Fierlinger, P. Geltenbort, M. Gupta, R. Henneck, S. Heule, M. Kasprzak, A. Knecht, M. Kuzniak, K. Kirch, M. Meier, A. Pichlmaier, R. Reiser, B. Theiler, O. Zimmer, G. Zsigmond, Nucl. Instrum. Methods B 260, 647 (2007).

29. M.H. Modi, G.S. Lodha, M.K. Tiwari, S.K. Rai, Nucl. Instrum. Methods B 239, 383 (2005).

30. Narziss, SINQ (PSI), http://kur.web.psi.ch/narziss/.

31. V.V. Nesvizhevsky et al., Nature 415, 297 (2002).

32. O. Filies, O. Böling, K. Grewer, J. Lekki, Appl. Surface Sci. 141, 357 (1999).

33. M. Makela, PhD Thesis, Virginia Polytechnic Institute and State University, Blacksburg, unpublished (2005). 